\title{
GENERALISED HUSIMI FUNCTIONS: ANALYTICITY AND INFORMATION CONTENT
}

\author{
D M APPLEBY \\ Department of Physics, Queen Mary and Westfield College, Mile End Rd, London
} E1 4NS, UK

(E-mail: D.M.Appleby@qmw.ac.uk)

\begin{abstract}
The analytic properties of a class of Generalised Husimi Functions are discussed, with particular reference to the problem of state reconstruction. The class consists of the subset of Wódkiewicz's operational probability distributions for which the filter reference state is a squeezed vacuum state. The fact that the function is analytic means that perfectly precise knowledge of its values over any small region of phase space provides enough information to reconstruct the density matrix. If, however, one only has imprecise knowledge of its values, then the amplification of statistical errors which occurs when one attempts to carry out the continuation seriously limits the amount of information which can be extracted. To take account of this fact a distinction is made between explicate, or experimentally accessible information, and information which is only present in implicate, experimentally inaccessible form. It is shown that an explicate description of various aspects of the system can be found localised on various 2 real dimensional surfaces in complexified phase space. In particular, the continuation of the function to the purely imaginary part of complexified phase space provides an explicate description of the Wigner function.
\end{abstract}




\section{INTRODUCTION}

The purpose of this paper is to discuss the analytic properties of a class of generalised Husimi functions, and the bearing that these properties have on the problem of state reconstruction.

The functions we consider may be written

$$
Q_{\lambda \theta}(x, p)=\frac{1}{\pi} \int d x^{\prime} d p^{\prime} \exp \left[-\frac{1}{\lambda^{2}}\left(x_{\theta}^{\prime}-x_{\theta}\right)^{2}-\lambda^{2}\left(p_{\theta}^{\prime}-p_{\theta}\right)^{2}\right] W\left(x^{\prime}, p^{\prime}\right)
$$

where $W$ is the Wigner function, and where the notation $x_{\theta}, p_{\theta}$ means

$$
\left(\begin{array}{l}
x_{\theta} \\
p_{\theta}
\end{array}\right)=\left(\begin{array}{cc}
\cos \theta & \sin \theta \\
-\sin \theta & \cos \theta
\end{array}\right)\left(\begin{array}{l}
x \\
p
\end{array}\right)
$$

The functions $Q_{\lambda \theta}$ belong to Wódkiewicz's class of operational probability distributions [1, 21. They are the distributions which result when the filter reference state is chosen to be an arbitrarily squeezed vacuum state [3]. They have previously been discussed by Halliwell [4], by Wünsche [5], and by Wünsche and Bužek [6].

In an earlier paper [7] we discussed the physical interpretation of these functions. We showed that $Q_{\lambda \theta}$ describes the probability distribution of measured values in the case when one makes a simultaneous, retrodictively optimal measurement (using homodyne detection [8, 9], for example) of $\hat{x}_{\theta}, \hat{p}_{\theta}$ to accuracies $\pm \frac{\lambda}{\sqrt{2}}, \pm \frac{1}{\sqrt{2}}$ respectively $\left[\hat{x}_{\theta}, \hat{p}_{\theta}\right.$ being defined in terms of $\hat{x}, \hat{p}$ by the obvious analogue of Eq. (2)]. This is a universal property: it only depends on the measurement being retrodictively optimal, and is otherwise independent of the details of the particular measuring process employed.

It is natural to ask: how much does one learn about the underlying quantum state from a knowledge of $Q_{\lambda \theta}$ ? Or, to put it another way: what is the information content of $Q_{\lambda \theta}$ ? The answer to this question depends on how accurately the function is known. If it is known with perfect accuracy, then it provides all the information necessary to reconstruct the density matrix [2, 6, 10, 11, 12, 13, 14, 15. However, the process of reconstruction is extremely sensitive to small variations in $Q_{\lambda \theta}$ 16]. Consequently, if one only has an approximate, experimental knowledge of the function, then it only provides a very limited amount of information regarding the state. One may express this by saying, that although the transformation by which one obtains $Q_{\lambda \theta}$ from the density matrix is invertible in point of mathematical principle, it is effectively non-invertible in point of experimental practice.

These statements apply to $Q_{\lambda \theta}(x, p)$ regarded as a function of two real variables. However, $Q_{\lambda \theta}(x, p)$ is in fact an analytic function of $x, p$ which continues to a holomorphic function defined on the whole of complexified phase space 10, 11, 12, 14, 17. Denote the continuation $Q_{\lambda \theta}^{\mathrm{c}}(x, p) . Q_{\lambda \theta}^{\mathrm{c}}(x, p)$ can itself be measured directly (by the method of quantum state sampling, for instance [9]). The question we address in the following is: how much information does one acquire by such measurements, either of the function as a whole, or of its restriction to various surfaces in complexified phase space?

Of course, if one has perfectly accurate knowledge of $Q_{\lambda \theta}^{\mathrm{c}}(x, p)$ on any small region of complexified phase space having at least one accumulation point then, because the function is holomorphic, that suffices to determine the function everywhere, and it consequently suffices to determine the density matrix. However, from our point of view this fact is not very interesting - for the reasons explained above. We are primarily interested, not in the information which can be extracted by some God-like being, endowed with perfectly accurate knowledge regarding the values of the function in some region, but rather with the information which is available to a real human experimenter, whose knowledge is necessarily imperfect. We are accordingly led to make a distinction between two different kinds of information, 
which we refer to as "explicate" and "implicate" (this terminology is based on the discussion in Bohm and Hiley [18]). Explicate information is information which is experimentally accessible. Implicate information is information which, though in some sense present, lies hidden in the fine details of the analytic structure, and is therefore only accessible if one has a perfectly accurate knowledge of the function. In these terms the question posed in the last paragraph may be rephrased: what is the explicate information content of the function $Q_{\lambda \theta}^{\mathrm{c}}(x, p)$ ? And: how is this information localised?

We begin in Section 2 by deriving what we shall refer to as the fundamental inversion formula. In the case $\theta=0$ this formula was obtained by Mehta and Sudarshan [10]. However, it appears to us that it has not received the attention it deserves. There do, of course, exist a number of other formulae expressing the density matrix in terms of the generalised Husimi function [2, 6, 13, 14, 19, 20, 21, 222. However, the formula derived in Section 2 differs from all of these in that it involves neither an integral nor an infinite sum. The formula shows, that modulo a Gaussian factor, the squeezed state density matrix elements (diagonal and offdiagonal) may actually be identified with the function $Q_{\lambda \theta}^{\mathrm{c}}$. It follows, that having an approximate knowledge of the values of $Q_{\lambda \theta}^{\mathrm{c}}(x, p)$ for $x, p$ arbitrary complex, is equivalent to knowing the density matrix, up to the same degree of approximation. In other words, $Q_{\lambda \theta}^{\mathrm{c}}$ (unlike $Q_{\lambda \theta}$ ) provides a fully explicate description of the density matrix.

In Section 3 we consider what happens if one attempts to use the analyticity to infer the complete function $Q_{\lambda \theta}^{\mathrm{c}}$ starting from a knowledge of the restricted function $Q_{\lambda \theta}$. We show that the errors grow exponentially fast as one moves away from the real plane. This means, that from an experimental point of view, the values which $Q_{\lambda \theta}^{\mathrm{c}}$ takes well away from the real plane are effectively independent of the values which it takes on the real plane. In Section 1 we go on to give a more precise definition of the concepts of explicate and implicate information.

The explicate information content of $Q_{\lambda \theta}^{\mathrm{c}}$ is not spread out evenly (so to speak). Rather, an explicate description of various different aspects of the system is to be found concentrated on various 2 real dimensional subspaces of the 4 real dimensional complexified phase space. In Sections 5 - 7 we discuss this phenomenon. In Section 5 we consider the problem of expressing the $x_{\phi}$-representation density matrix elements in terms of $Q_{\lambda \theta}^{\mathrm{c}}$. In Section 6 we consider the problem of expressing the function $Q_{\lambda \theta}$ given by one choice of the parameters $\lambda, \theta$ in terms of the function $Q_{\lambda^{\prime} \theta^{\prime}}^{\mathrm{c}}$ given by some other choice. In Section 7 we consider the problem of expressing other phase space distributions in terms of $Q_{\lambda \theta}^{\mathrm{c}}$. In particular, we show that the continuation to the purely imaginary part of complexified phase space contains an explicate description of the Wigner function. We also show that the existence and regularity of these distributions depends on the growth of $Q_{\lambda \theta}^{\mathrm{c}}$ as one moves away from the real plane.

Finally, in Section 8 we show how the properties of $Q_{\lambda \theta}$ can be used to give an interesting illustration of the concept of complementarity.

\section{The Fundamental Inversion Formula}

We begin by fixing some notation. Let $\hat{a}_{\lambda \theta}$ be the annihilation operator defined by

$$
\hat{a}_{\lambda \theta}=\frac{1}{\sqrt{2}}\left(\frac{1}{\lambda} \hat{x}_{\theta}+i \lambda \hat{p}_{\theta}\right)
$$

where $\hat{x}_{\theta}, \hat{p}_{\theta}$ are the operators defined by the obvious analogue of Eq. (2). Let $|n\rangle_{\lambda \theta}$ be a normalised eigenstate of the number operator $\hat{a}_{\lambda \theta}^{\dagger} \hat{a}_{\lambda \theta}$ with eigenvalue $n$, and 
let $\left|(x, p)_{\lambda \theta}\right\rangle$ be the squeezed state defined by

$$
\left|(x, p)_{\lambda \theta}\right\rangle=\exp \left(-\frac{1}{2}|z|^{2}\right) \sum_{n=0}^{\infty} \frac{z^{n}}{\sqrt{n !}}|n\rangle_{\lambda \theta}
$$

where $z=\frac{1}{\sqrt{2}}\left(\frac{1}{\lambda} x_{\theta}+i \lambda p_{\theta}\right)$. Then the generalised Husimi function can be written

$$
Q_{\lambda \theta}(x, p)=\frac{1}{2 \pi}\left\langle(x, p)_{\lambda \theta}|\hat{\rho}|(x, p)_{\lambda \theta}\right\rangle
$$

where $\hat{\rho}$ is the density matrix. More generally one may define, for any operator $\hat{A}$,

$$
A_{\lambda \theta}(x, p)=\left\langle(x, p)_{\lambda \theta}|\hat{A}|(x, p)_{\lambda \theta}\right\rangle
$$

We will refer to this function as the generalised Husimi transform of $\hat{A}$.

The problem of reconstructing the density matrix from an exact knowledge of $Q_{\lambda \theta}$, and more generally the problem of reconstructing an operator $\hat{A}$ from an exact knowledge of $A_{\lambda \theta}$, comes down to the problem of using the diagonal matrix elements in a squeezed state representation to reconstruct the off-diagonal elements. The possibility of such a reconstruction may, at first sight, seem rather surprising. It is certainly not guaranteed just by the over-completeness of the set of squeezed states (one can easily think of over-complete sets which do not have this property). What makes it possible is the analyticity of the transform.

There are, in fact, two different kinds of analyticity which are relevant. In the case of a pure state Prugovečki [12] has used the analyticity of the Fock-Bargmann wave function 23, 24 to show that the Husimi function completely determines the state. However, it is not clear how to extend Prugovečki's argument to deal with the case of an arbitrary density matrix. Also, Prugovečki does not give a formula for actually carrying out the inversion.

Rather more useful is the result obtained by Mehta and Sudarshan [10]. Since Mehta and Sudarshan do not consider the case $\theta \neq 0$, and since we feel that the result deserves to be more widely known, we give the argument here.

In view of Eqs. (3) and (4) we have

$$
A_{\lambda \theta}(x, p)=\exp \left(-|z|^{2}\right) \sum_{n, m=0}^{\infty} \frac{z^{* n} z^{m}}{\sqrt{n ! m !}}{ }_{\lambda \theta}\langle n|\hat{A}| m\rangle_{\lambda \theta}
$$

Until now we have been assuming that $x$ and $p$ are both real. However, we can still make sense of this formula for complex $x, p$. Define

$$
z_{ \pm}=\frac{1}{\sqrt{2}}\left(\frac{1}{\lambda} x_{\theta} \pm i \lambda p_{\theta}\right)
$$

If $x, p$ are allowed to be complex then $z_{ \pm}$are independent complex variables. Consider the expression

$$
A_{\lambda \theta}^{\mathrm{c}}(x, p)=\exp \left(-z_{-} z_{+}\right) \sum_{n, m=0}^{\infty} \frac{z_{-}^{n} z_{+}^{m}}{\sqrt{n ! m !}} \lambda \theta|n| \hat{A}|m\rangle_{\lambda \theta}
$$

It is straightforward to show [11] that the series on the right hand side of this equation converges if there exists a positive constant $K$, and a constant $\alpha$ in the range $0 \leq \alpha<1$, such that

$$
\| \hat{A}|n\rangle_{\lambda \theta} \| \leq\left(K n^{\alpha}\right)^{n}
$$

for all $n$. In that case Eq. (6) gives the continuation of $A_{\lambda \theta}$ to a holomorphic function defined on the whole of $\mathbb{C}^{2}$. 
Let us now see how we can use the continuation to invert the transform. Consider the off-diagonal matrix element $\left\langle\left(x_{1}, p_{1}\right)_{\lambda \theta}|\hat{A}|\left(x_{2}, p_{2}\right)_{\lambda \theta}\right\rangle$. We have

$$
\begin{aligned}
& \left\langle\left(x_{1}, p_{1}\right)_{\lambda \theta}|\hat{A}|\left(x_{2}, p_{2}\right)_{\lambda \theta}\right\rangle \\
& \quad=\exp \left[-\frac{1}{2}\left(\left|z_{1}\right|^{2}+\left|z_{2}\right|^{2}\right)\right] \sum_{n, m=0}^{\infty} \frac{z_{1}^{* n} z_{2}^{m}}{\sqrt{n ! m !}} \lambda\langle n|\hat{A}| m\rangle_{\lambda}
\end{aligned}
$$

where

$$
z_{r}=\frac{1}{\sqrt{2}}\left(\frac{1}{\lambda} x_{r \theta}+i \lambda p_{r \theta}\right) \quad r=1,2
$$

The points $\left(x_{1}, p_{1}\right)$ and $\left(x_{2}, p_{2}\right)$ both belong to $\mathbb{R}^{2}$. We now use them to define a single point $(x, p)$ in complexified phase space

$$
\begin{aligned}
& x=\frac{1}{2}\left(x_{1}+x_{2}\right)+\frac{i}{2} \sinh 2 \eta \sin 2 \theta\left(x_{1}-x_{2}\right)-\frac{i}{2}(\cosh 2 \eta+\sinh 2 \eta \cos 2 \theta)\left(p_{1}-p_{2}\right) \\
& p=\frac{1}{2}\left(p_{1}+p_{2}\right)+\frac{i}{2}(\cosh 2 \eta-\sinh 2 \eta \cos 2 \theta)\left(x_{1}-x_{2}\right)-\frac{i}{2} \sinh 2 \eta \sin 2 \theta\left(p_{1}-p_{2}\right)
\end{aligned}
$$

where we have set $\eta=\ln \lambda$. We have

$$
\begin{aligned}
& x_{\theta}=\cos \theta x+\sin \theta p=\frac{1}{2}\left(x_{1 \theta}+x_{2 \theta}\right)-\frac{i \lambda^{2}}{2}\left(p_{1 \theta}-p_{2 \theta}\right) \\
& p_{\theta}=-\sin \theta x+\cos \theta p=\frac{1}{2}\left(p_{1 \theta}+p_{2 \theta}\right)+\frac{i}{2 \lambda^{2}}\left(x_{1 \theta}-x_{2 \theta}\right)
\end{aligned}
$$

Consequently

$$
z_{+}=z_{2} \quad \text { and } \quad z_{-}=z_{1}^{*}
$$

where $z_{ \pm}$are the quantities defined by Eq. (5). Inserting these relations in Eq. (7) and comparing with Eq. (6) we deduce

$$
\left\langle\left(x_{1}, p_{1}\right)_{\lambda \theta}|\hat{A}|\left(x_{2}, p_{2}\right)_{\lambda \theta}\right\rangle=\exp \left[-\frac{1}{2}\left(\left|z_{1}\right|^{2}+\left|z_{2}\right|^{2}\right)+z_{1}^{*} z_{2}\right] A_{\lambda \theta}^{\mathrm{c}}(x, p)
$$

which can alternatively be written

$$
A_{\lambda \theta}^{\mathrm{c}}(x, p)=\frac{\left\langle\left(x_{1}, p_{1}\right)_{\lambda \theta}|\hat{A}|\left(x_{2}, p_{2}\right)_{\lambda \theta}\right\rangle}{\left\langle\left(x_{1}, p_{1}\right)_{\lambda \theta} \mid\left(x_{2}, p_{2}\right)_{\lambda \theta}\right\rangle}
$$

Eq. (8) relates the off-diagonal matrix elements to the analytic continuation of the diagonal elements. We will refer to it as the fundamental inversion formula.

If $\theta=0$ and $\lambda=1$, and if $\hat{A}$ is a density matrix, then the expression on the left hand side is the $Q$-function, continued to complex values of $x$ and $p$ (and modulo a factor $\frac{1}{2 \pi}$ ); while the expression on the right hand side is the $R$-representation [25] [modulo a factor $\left.\exp \left(-z_{1}^{*} z_{2}\right)\right]$. We see, therefore, that the $R$-representation is essentially the same thing as the analytic continuation of the $Q$-function-as was originally noted by Mehta and Sudarshan [10].

There do, of course, exist other ways of inverting the transform [2, 6, 13, 14, 19, 20, 21, 22. However, these methods all involve, either an integral, or else the calculation of an infinite sum. The equation just derived is more straightforward. It shows, that in order to invert the generalised Husimi transform, all that one has to do is to perform the analytic continuation, and then to multiply by a Gaussian factor.

Eq. (8) also shows that the function $Q_{\lambda \theta}^{\mathrm{c}}$ can be measured directly, by the method of quantum state sampling [9]. 


\section{The Physical Significance of the Analyticity}

Eq. (8) shows that an exact specification of $Q_{\lambda \theta}(x, p)$ for real $x, p$ suffices to determine the density matrix. Actually, a very much stronger statement is true: once $Q_{\lambda \theta}^{\text {c }}$ is exactly known on a region of complexified phase space having at least one accumulation point, then that suffices to determine the function everywhere, and it consequently suffices to determine the density matrix. Alternatively, an exact knowledge of the function together with all its derivatives at any one point suffices to completely determine it at every other point (as has been stressed by Wünsche and Bužek [6]). The function might therefore be compared with a hologram: information sufficient to reconstruct the whole picture is folded into every little piece of it.

$Q_{\lambda \theta}$ is a very intricately structured object. In particular, the analyticity means that there exist subtle connections between the probability of finding the system in one part of phase space, and the probability of finding it another. As an illustration of this point, specialise to the case $\theta=0$, and consider the following expression 11] for the quantity ${ }_{\lambda 0}\langle n|\hat{\rho}| n\rangle_{\lambda 0}\left(|n\rangle_{\lambda 0}\right.$ being the number state defined at the beginning of the last section):

$$
{ }_{\lambda 0}\langle n|\hat{\rho}| n\rangle_{\lambda 0}=\left.2 \pi \sum_{r=0}^{n} \frac{n !}{(n-r) !(r !)^{2}} \frac{1}{2^{r}}\left[\left(\lambda^{2} \frac{\partial^{2}}{\partial x^{2}}+\frac{1}{\lambda^{2}} \frac{\partial^{2}}{\partial p^{2}}\right)^{r} Q_{\lambda 0}(x, p)\right]\right|_{x=p=0}
$$

${ }_{\lambda 0}\langle n|\hat{\rho}| n\rangle_{\lambda 0}$ is the probability of finding the system in the state $|n\rangle_{\lambda 0}$. It therefore tells us something about the probability of finding the system in the vicinity of the surface $\frac{1}{\lambda^{2}} x^{2}+\lambda^{2} p^{2}=2 n+1$. Yet in order to calculate the probability we have to evaluate $Q_{\lambda 0}$ and its derivatives, not in the vicinity of this surface, but at the origin.

The feature just evinced could be regarded as a kind of non-locality. It is somewhat reminiscent of the kind of non-locality that is manifested by a violation of the Bell inequalities [26].

Bohm and Hiley [18] place much emphasis on what, following Bohr [27], they describe as a property of "wholeness" possessed by quantum mechanical systems. In their discussion of this feature Bohm and Hiley also make use of a hologram analogy. They argue that the property of wholeness does not essentially depend on assumptions peculiar to "hidden-variables" interpretations of quantum mechanics, such as the Bohm interpretation. On the contrary, they maintain that it appears, in one guise or another, whatever the interpretational scheme one adopts (it appears in the Copenhagen interpretation favoured by Bohr, for example). The analyticity of the function $Q_{\lambda \theta}^{\mathrm{c}}$ could be regarded as another illustration of these ideas.

This phenomenon of "wholeness" is certainly intriguing. However, it is also elusive. The non-local connections in the Bohm interpretation cannot be used for faster-than-light signalling. The practical significance of the kind of non-locality exemplified by Eq. (9) is also very limited. It critically depends on $Q_{\lambda \theta}^{\mathrm{c}}$ being known with perfect exactness. However, $Q_{\lambda \theta}^{\mathrm{c}}$ could in fact only ever be measured up to a certain non-zero error, which seriously restricts the usefulness of the analyticity.

To see this, consider the problem of calculating $Q_{\lambda \theta}^{\mathrm{c}}$ at a point $(x+\eta, p+\zeta)$ using the expansion

$$
Q_{\lambda \theta}^{\mathrm{c}}(x+\eta, p+\zeta)=\sum_{n, m=0}^{\infty} \frac{\eta^{n} \zeta^{m}}{n ! m !} \frac{\partial^{n+m}}{\partial x^{n} \partial p^{m}} Q_{\lambda \theta}^{\mathrm{c}}(x, p)
$$

Suppose that the derivatives appearing in this sum have been measured independently. For the sake of example, suppose that the error in the measurement of $\frac{\partial^{n+m}}{\partial x^{n} \partial p^{m}} Q_{\lambda \theta}^{\mathrm{c}}$ is $\pm \lambda^{m-n} \sigma$, where $\sigma$ is a dimensionless constant. Let $\Delta Q$ be the error 
in $Q_{\lambda \theta}^{\mathrm{c}}(x+\eta, p+\zeta)$, as calculated using the above formula. Then

$$
\Delta Q=\sigma\left(\sum_{n, m=0}^{\infty} \frac{|\eta|^{2 n}|\zeta|^{2 m}}{(n ! m !)^{2}} \lambda^{2(m-n)}\right)^{\frac{1}{2}}=\sigma\left[I_{0}\left(2 \lambda^{-1}|\eta|\right) I_{0}(2 \lambda|\zeta|)\right]^{\frac{1}{2}}
$$

where $I_{0}$ is a Bessel function of imaginary argument [28]. Asymptotically 28]

$$
\Delta Q \sim \frac{1}{2} \sigma\left(\pi^{2}|\eta||\zeta|\right)^{-\frac{1}{4}} \exp \left(\lambda^{-1}|\eta|+\lambda|\zeta|\right)
$$

It can be seen that the error grows exponentially as one moves away from the point $(x, p)$. If, instead of direct measurements of the derivatives, one only had imprecise information regarding the values of the function itself in some small region surrounding the point $(x, p)$, from which the values of the derivatives had to be estimated, then one would expect the error to grow even more rapidly.

We see that the situation is not really so different from the case of a function which is not analytic. Given an experimental determination of the function in some region, one can use the analyticity to infer something about its behaviour a small distance outside that region. However, one cannot go very far before the data is swamped by the errors.

Although they are not independent in point of mathematical principle, it may be said that the values which $Q_{\lambda \theta}^{\mathrm{c}}$ takes in well-separated regions of complexified phase space are effectively independent in point of experimental practice.

The situation here could be compared with the case of a classically chaotic deterministic system. In principle the state of such a system at any one time suffices to completely determine its state at every other time. However, the practical usefulness of this fact is very limited. The information is in some sense present, but mostly not in a form which it is possible to access.

\section{Explicate V. Implicate Information Content}

In view of the discussion in the last section we are led to distinguish between two different kinds of information which, following Bohm and Hiley [18], we will refer to as "explicate" and "implicate" (although we do not use these words in quite the same sense as Bohm and Hiley). Explicate information is information which approximately survives the process of making inexact measurements. Implicate information is information which is experimentally inaccessible.

In order to make these notions a little more precise, consider a physical quantity which is given by an expression of the form

$$
\int_{D} f(x, p) Q_{\lambda \theta}^{\mathrm{c}}(x, p) d \mu
$$

In this expression the domain of integration $D$ might be a 4 real dimensional volume in complexified phase space, or it might be a lower dimensional surface (such as the real plane). $f$ might be an ordinary function, or it might only be defined in a distributional sense.

Suppose that $f$ is an ordinary function. In that case the error in the integral is of a comparable order to the error in $Q_{\lambda \theta}^{\mathrm{c}}$. The same is true for certain, not too singular distributions. It is true if $f$ is a $\delta$ function, for example. In such a case we will say that $Q_{\lambda \theta}^{\mathrm{c}}$, restricted to the region $D$, contains explicate information regarding the physical quantity in question.

Suppose, on the other hand, that $f$ is a very singular distribution. Suppose, for example, that $f$ involves many high order derivatives of the $\delta$ function. In that case, although the integral may still be of some theoretical interest, it cannot easily be used to make practical calculations based on inexact experimental data concerning 
the function $Q_{\lambda \theta}^{\mathrm{c}}$. We will say that $Q_{\lambda \theta}^{\mathrm{c}}$, restricted to the region $D$, only contains implicate information about the physical quantity in question.

The distinction between "explicate" and "implicate" is qualitative, and it should not be taken in too absolute a sense. For instance, one cannot entirely rule out the possibility of measuring all the quantities on the right hand side of Eq. (9), and using them to make a good estimate of ${ }_{\lambda 0}\langle n|\hat{\rho}| n\rangle_{\lambda 0}$. However, it would obviously be very difficult if $n$ is large.

As an illustration of these concepts, consider the problem of calculating the expectation value of an operator $\hat{A}$. Using Eq. (\$) we have

$$
\begin{aligned}
\operatorname{Tr}(\hat{A} \hat{\rho}) & =\frac{1}{4 \pi^{2}} \int d x_{1} d p_{1} d x_{2} d p_{2}\left\langle\left(x_{1}, p_{1}\right)_{\lambda \theta}|\hat{A}|\left(x_{2}, p_{2}\right)_{\lambda \theta}\right\rangle\left\langle\left(x_{2}, p_{2}\right)_{\lambda \theta}|\hat{\rho}|\left(x_{1}, p_{1}\right)_{\lambda \theta}\right\rangle \\
& =\frac{2}{\pi} \int d^{2} x d^{2} p \exp \left(-\frac{2}{\lambda^{2}} x_{\mathrm{I} \theta}^{2}-2 \lambda^{2} p_{\mathrm{I} \theta}^{2}\right) A_{\lambda \theta}^{\mathrm{c}}\left(x^{*}, p^{*}\right) Q_{\lambda \theta}^{\mathrm{c}}(x, p)
\end{aligned}
$$

In this expression $x_{\mathrm{I}}, p_{\mathrm{I}}$ denote the imaginary parts of the complex variables $x$, $p . x_{\mathrm{I} \theta}, p_{\mathrm{I} \theta}$ are defined in terms of $x_{\mathrm{I}}, p_{\mathrm{I}}$ as in Eq. (2). The integral is taken over the whole of complexified phase space. It can be seen that $Q_{\lambda \theta}^{\mathrm{c}}$ contains explicate information about $\langle\hat{A}\rangle$ whenever $A_{\lambda \theta}^{\mathrm{c}}$ is defined as an ordinary function-which is the case for most operators of physical interest.

Suppose, on the other hand, that one wants to calculate $\langle\hat{A}\rangle$ using only the values of the restricted function $Q_{\lambda \theta}$. Inspection of Eq. (10) shows, that due to the presence of the Gaussian factor, the integrand will be strongly peaked about the real plane, provided that $A_{\lambda \theta}^{\mathrm{c}}\left(x^{*}, p^{*}\right)$ does not grow too rapidly with increasing $x_{\mathrm{I}}$, $p_{\mathrm{I}}$. In that case we may expand $A_{\lambda \theta}^{\mathrm{c}}\left(x^{*}, p^{*}\right)$, and $Q_{\lambda \theta}^{\mathrm{c}}(x, p)$ about the point $\left(x_{\mathrm{R}}, p_{\mathrm{R}}\right)$ (where $x_{\mathrm{R}}, p_{\mathrm{R}}$ denote the real parts of $x, p$ ):

$$
\begin{aligned}
\operatorname{Tr}(\hat{A} \hat{\rho})=\frac{2}{\pi} \sum_{n, n^{\prime}, m, m^{\prime}=0}^{\infty} & \int d^{2} x d^{2} p \exp \left(-\frac{2}{\lambda^{2}} x_{\mathrm{I} \theta}^{2}-2 \lambda^{2} p_{\mathrm{I} \theta}^{2}\right) \frac{(-1)^{n+m} i^{n+n^{\prime}+m+m^{\prime}}}{n ! n^{\prime} ! m ! m^{\prime} !} \\
& \times x_{\mathrm{I}}^{n+n^{\prime}} p_{\mathrm{I}}^{m+m^{\prime}} \frac{\partial^{n+m}}{\partial x_{\mathrm{R}}^{n} \partial p_{\mathrm{R}}^{m}} A_{\lambda \theta}\left(x_{\mathrm{R}}, p_{\mathrm{R}}\right) \frac{\partial^{n^{\prime}+m^{\prime}}}{\partial x_{\mathrm{R}}^{n^{\prime}} \partial p_{\mathrm{R}}^{m^{\prime}}} Q_{\lambda \theta}\left(x_{\mathrm{R}}, p_{\mathrm{R}}\right)
\end{aligned}
$$

One finds

$$
\operatorname{Tr}(\hat{A} \hat{\rho})=\int d x_{\mathrm{R}} d p_{\mathrm{R}} A_{\lambda \theta}^{\mathrm{a}}\left(x_{\mathrm{R}}, p_{\mathrm{R}}\right) Q_{\lambda \theta}\left(x_{\mathrm{R}}, p_{\mathrm{R}}\right)
$$

where $A_{\lambda \theta}^{\mathrm{a}}$ is the generalised anti-Husimi transform [20, 21], defined by

$$
A_{\lambda \theta}^{\mathrm{a}}\left(x_{\mathrm{R}}, p_{\mathrm{R}}\right)=\exp \left[-\frac{1}{2}\left(\lambda^{2} \frac{\partial^{2}}{\partial x_{\mathrm{R} \theta}^{2}}+\frac{1}{\lambda^{2}} \frac{\partial^{2}}{\partial p_{\mathrm{R} \theta}^{2}}\right)\right] A_{\lambda \theta}\left(x_{\mathrm{R}}, p_{\mathrm{R}}\right)
$$

There are, of course, easier ways [2] of deriving Eq. (11). The advantage of the derivation just indicated is that it brings out the connection between Eqs. (10) and (11). It also brings out the fact that the existence of $A_{\lambda \theta}^{\mathrm{a}}$ as an ordinary function depends on the growth of $A_{\lambda \theta}^{\mathrm{c}}$ as one moves away from the real plane. We will return to this point in Section 1 .

If $A_{\lambda \theta}^{\mathrm{a}}$ exists as an ordinary function, or as a not too singular distribution, then $Q_{\lambda \theta}$ provides us with explicate information regarding $\langle\hat{A}\rangle$. However, $A_{\lambda \theta}^{\mathrm{a}}$ is in fact often very singular. The conclusion is, therefore, that whereas $Q_{\lambda \theta}^{\mathrm{c}}$ provides us with an explicate description of the quantum state, the description provided by $Q_{\lambda \theta}$ is, for the most part, only implicate.

$Q_{\lambda \theta}$ is the probability distribution describing the outcome of a retrodictively optimal determination of phase space location $[7]$. It might therefore be compared with the function $|\langle x \mid \psi\rangle|^{2}$. The values which $Q_{\lambda \theta}^{\mathrm{c}}$ takes away from the real plane 
provide us with the additional, explicate information needed to calculate the expectation value of an arbitrary observable. They might therefore be compared with the phase of the function $\langle x \mid \psi\rangle$.

\section{Connection with the $x_{\phi}$-Representation Matrix Elements}

The advantage of Eq. (11) is that it only requires us to integrate over two real variables, unlike Eq. (10) which requires us to integrate over four. The disadvantage is that $A_{\lambda \theta}^{\mathrm{a}}$ is often extremely singular. It is natural to ask whether one might do better by integrating over some other surface, instead of the real plane. In the next three sections we investigate this question. We begin by identifying a surface, having two real dimensions, which contains an explicate description of the matrix elements

$$
{ }_{\phi}\left\langle x_{1}|\hat{\rho}| x_{2}\right\rangle_{\phi}
$$

(where $|x\rangle_{\phi}$ is an eigenket of $\hat{x}_{\phi}$ with eigenvalue $x$ ). The result we derive is a generalisation of the one proved by Davidović and Lalović [14].

It is readily shown [3, 9]

$$
\begin{aligned}
{ }_{\phi}\left\langle x^{\prime} \mid(x, p)_{\lambda \theta}\right\rangle= & \left\langle x^{\prime} \mid\left(x_{\phi}, p_{\phi}\right)_{\lambda \delta}\right\rangle \\
= & \left(\frac{e^{-i \delta}}{\sqrt{\pi}\left(\lambda \cos \delta-i \lambda^{-1} \sin \delta\right)}\right)^{\frac{1}{2}} \\
& \quad \times \exp \left[-\frac{\lambda^{-1} \cos \delta-i \lambda \sin \delta}{2\left(\lambda \cos \delta-i \lambda^{-1} \sin \delta\right)}\left(x^{\prime}-x_{\phi}\right)^{2}+i p_{\phi} x^{\prime}-\frac{1}{2} p_{\phi} x_{\phi}\right]
\end{aligned}
$$

where $\delta=\theta-\phi$. Consequently

$$
\begin{aligned}
Q_{\lambda \theta}(x, p)=\frac{1}{\pi^{\frac{3}{2}} \sqrt{b}} \int d x^{\prime} d y^{\prime} \exp [ & -\frac{1}{b}\left(\left(x^{\prime}-x_{\phi}\right)^{2}+y^{\prime 2}\right) \\
& \left.-\frac{2 i c}{b} y^{\prime}\left(x^{\prime}-x_{\phi}\right)+2 i p_{\phi} y^{\prime}\right]{ }_{\phi}\left\langle x^{\prime}-y^{\prime}|\hat{\rho}| x^{\prime}+y^{\prime}\right\rangle_{\phi}
\end{aligned}
$$

where

$$
\begin{aligned}
& b=\lambda^{2} \cos ^{2} \delta+\lambda^{-2} \sin ^{2} \delta \\
& c=-\left(\lambda^{2}-\lambda^{-2}\right) \sin \delta \cos \delta
\end{aligned}
$$

Continuing to complex values of $x$ and $p$ we find

$$
\begin{aligned}
& \exp \left(-b u^{2}\right) Q_{\lambda \theta}^{\mathrm{c}}(-v \sin \phi+i u d, v \cos \phi+i u f) \\
&=\frac{1}{\pi^{\frac{3}{2}} \sqrt{b}} \int d x d y \exp [2 i(u x+v y)] \\
& \times \exp \left[-\frac{1}{b}\left(x^{2}+2 i c x y+y^{2}\right)\right]_{\phi}\langle x-y|\hat{\rho}| x+y\rangle_{\phi}
\end{aligned}
$$

where

$$
\begin{aligned}
& d=b \cos \phi+c \sin \phi=\lambda^{2} \cos \theta \cos \delta+\lambda^{-2} \sin \theta \sin \delta \\
& f=b \sin \phi-c \cos \phi=\lambda^{2} \sin \theta \cos \delta-\lambda^{-2} \cos \theta \sin \delta
\end{aligned}
$$


Inverting the Fourier transform gives

$$
\begin{aligned}
\exp \left[-\frac{1}{b}\left(x^{2}+2 i c x y+y^{2}\right)\right]_{\phi}\langle x-y|\hat{\rho}| x+y\rangle_{\phi} \\
=\left(\frac{b}{\pi}\right)^{\frac{1}{2}} \int d u d v \exp [-2 i(u x+v y)] \\
\times \exp \left(-b u^{2}\right) Q_{\lambda \theta}^{\mathrm{c}}(-v \sin \phi+i u d, v \cos \phi+i u f)
\end{aligned}
$$

which is the desired result.

By essentially the same argument one can derive an analogous formula relating the $x_{\phi}$-representation matrix elements of an arbitrary operator $\hat{A}$ to the function $A_{\lambda \theta}^{\mathrm{c}}$.

\section{6. $Q_{\lambda^{\prime} \theta^{\prime}}$ IN TERMS OF $Q^{\mathrm{c}}$}

Using similar means one may obtain an explicate description of the function $Q_{\lambda \theta}$ by integrating $Q_{\lambda^{\prime} \theta^{\prime}}^{\mathrm{c}}$ over a surface having 2 real dimensions. However, the general formula is rather complicated. We will therefore confine ourselves to showing how $Q_{\lambda \theta}$ may be expressed in terms of the analytic continuation of the $Q$-function, $Q^{\mathrm{c}}=Q_{10}^{\mathrm{c}}$.

Suppose that $\lambda<1$. Eq. (14) gives

$$
\begin{aligned}
& \exp \left(-\lambda^{-2} u^{2}\right) Q_{\lambda \theta}^{\mathrm{c}}(-v \sin \theta+i u \cos \theta, v \cos \theta+i u \sin \theta) \\
& \quad=\frac{1}{\pi^{\frac{3}{2}} \lambda} \int d x d y \exp \left[2 i\left(\lambda^{-2} u x+v y\right)\right] \exp \left[-\lambda^{-2}\left(x^{2}+y^{2}\right)\right]_{\theta}\langle x-y|\hat{\rho}| x+y\rangle_{\theta}
\end{aligned}
$$

while Eq. 15) gives

$$
\begin{aligned}
\exp \left[-\left(x^{2}+y^{2}\right)\right]_{\theta}\langle x-y|\hat{\rho}| x+y\rangle_{\theta} & \\
=\frac{1}{\sqrt{\pi}} \int d u^{\prime} d v^{\prime} \exp \left[-2 i\left(u^{\prime} x\right.\right. & \left.\left.+v^{\prime} y\right)\right] \exp \left(-u^{2}\right) \\
& \times Q^{\mathrm{c}}\left(-v^{\prime} \sin \theta+i u^{\prime} \cos \theta, v^{\prime} \cos \theta+i u^{\prime} \sin \theta\right)
\end{aligned}
$$

Combining these formulae we obtain

$$
\begin{aligned}
& \exp \left[-\frac{1}{1-\lambda^{2}} x_{\theta}^{2}\right] Q_{\lambda \theta}(x, p) \\
& =\frac{\lambda}{\pi\left(1-\lambda^{2}\right)} \int d u^{\prime} d v^{\prime} \exp \left[-\frac{2 i}{1-\lambda^{2}} u^{\prime} x_{\theta}-\frac{1}{\lambda^{-2}-1}\left(v^{\prime}-p_{\theta}\right)^{2}\right] \\
& \quad \times \exp \left[-\frac{1}{1-\lambda^{2}} u^{\prime 2}\right] Q^{\mathrm{c}}\left(-v^{\prime} \sin \theta+i u^{\prime} \cos \theta, v^{\prime} \cos \theta+i u^{\prime} \sin \theta\right)
\end{aligned}
$$

If $\lambda>1$ we have

$$
\begin{aligned}
& \exp \left[-\frac{1}{1-\lambda^{-2}} p_{\theta}^{2}\right] Q_{\lambda \theta}(x, p) \\
& =\frac{\lambda^{-1}}{\pi\left(1-\lambda^{-2}\right)} \int d u^{\prime} d v^{\prime} \exp \left[\frac{2 i}{1-\lambda^{-2}} u^{\prime} p_{\theta}-\frac{1}{\lambda^{2}-1}\left(v^{\prime}-x_{\theta}\right)^{2}\right] \\
& \quad \times \exp \left[-\frac{1}{1-\lambda^{-2}} u^{\prime 2}\right] Q^{\mathrm{c}}\left(v^{\prime} \cos \theta+i u^{\prime} \sin \theta, v^{\prime} \sin \theta-i u^{\prime} \cos \theta\right)
\end{aligned}
$$

$Q$ describes the result 7 of measuring $x_{\theta}, p_{\theta}$ both to a retrodictive accuracy of $\pm \frac{1}{\sqrt{2}}$. Eq. (16) shows that continuing to one part of complexified phase space gives a more accurate explicate description of $x_{\theta}$. Eq. (17) shows that continuing to a 
different part of complexified phase space gives a more accurate explicate description of $p_{\theta}$. An explicate description of the result of every possible measurement is to be found somewhere in complexified phase space.

Analogous formulae hold for the generalised Husimi transform of an arbitrary operator $\hat{A}$.

\section{The Generalised $s$-Transform}

The generalised $s$-transform of an operator $\hat{A}$ is defined by

$$
A_{\lambda \theta}^{(s)}(x, p)=\frac{1}{2 \pi} \int d x^{\prime} d p^{\prime} \exp \left[i\left(p x^{\prime}-x p^{\prime}\right)+\frac{s}{4}\left(\lambda^{2} p_{\theta}^{\prime 2}+\lambda^{-2} x_{\theta}^{\prime 2}\right)\right] \operatorname{Tr}\left(\hat{D}_{x^{\prime} p^{\prime}} \hat{A}\right)
$$

where $\hat{D}_{x p}=\exp [i(p \hat{x}-x \hat{p})]$ is the displacement operator.

If $s>-1$ it is straightforward to show

$$
\begin{gathered}
\hat{A}_{\lambda \theta}(x, p)=\frac{1}{\pi(1+s)} \int d x^{\prime} d p^{\prime} \exp \left[-\frac{1}{(1+s)}\left(\lambda^{-2}\left(x_{\theta}^{\prime}-x_{\theta}\right)^{2}+\lambda^{2}\left(p_{\theta}^{\prime}-p_{\theta}\right)^{2}\right)\right] \\
\times A_{\lambda \theta}^{(s)}\left(x^{\prime}, p^{\prime}\right)
\end{gathered}
$$

This formula can be inverted using

$$
A_{\lambda \theta}^{(s)}(x, p)=\exp \left[-\frac{1+s}{4}\left(\lambda^{2} \frac{\partial^{2}}{\partial x_{\theta}^{2}}+\frac{1}{\lambda^{2}} \frac{\partial^{2}}{\partial p_{\theta}^{2}}\right)\right] A_{\lambda \theta}(x, p)
$$

It is to be observed, however, that the expression on the right hand side of Eq. (19) contains derivatives of all orders, which means it is exploiting information that is only present in implicate form. If one wants a formula which only depends on information present in explicate form it is necessary to make use of the analytic continuation.

Continuing to imaginary values of $x$ and $p$ in Eq. (18) gives, after rearranging,

$$
\begin{aligned}
\exp & {\left[-\frac{1}{1+s}\left(\lambda^{-2} x^{2}+\lambda^{2} p^{2}\right)\right] A_{\lambda \theta}^{\mathrm{c}}\left(i x_{-\theta}, i p_{-\theta}\right) } \\
=\frac{1}{\pi(1+s)} \int d x^{\prime} d p^{\prime} & \exp \left[\frac{2 i}{(1+s)}\left(\lambda^{-2} x x^{\prime}+\lambda^{2} p p^{\prime}\right)\right] \\
\times & \exp \left[-\frac{1}{1+s}\left(\lambda^{-2} x^{\prime 2}+\lambda^{2} p^{\prime 2}\right)\right] A_{\lambda \theta}^{(s)}\left(x_{-\theta}^{\prime}, p_{-\theta}^{\prime}\right)
\end{aligned}
$$

Inverting the Fourier transform and changing the variables of integration gives

$$
\begin{aligned}
& \exp \left[-\frac{1}{1+s}\left(\lambda^{-2} x_{\theta}^{2}+\lambda^{2} p_{\theta}^{2}\right)\right] A_{\lambda \theta}^{(s)}(x, p) \\
&=\frac{1}{\pi(1+s)} \int d x^{\prime} d p^{\prime} \exp \left[-\frac{2 i}{(1+s)}\left(\lambda^{-2} x_{\theta} x_{\theta}^{\prime}+\lambda^{2} p_{\theta} p_{\theta}^{\prime}\right)\right] \\
& \times \exp \left[-\frac{1}{1+s}\left(\lambda^{-2} x_{\theta}^{\prime 2}+\lambda^{2} p_{\theta}^{\prime 2}\right)\right] A_{\lambda \theta}^{\mathrm{c}}\left(i x^{\prime}, i p^{\prime}\right)
\end{aligned}
$$

If $s=0$ and $\hat{A}$ is the density matrix this becomes

$$
\begin{aligned}
& \exp \left[-\left(\lambda^{-2} x_{\theta}^{2}+\lambda^{2} p_{\theta}^{2}\right)\right] W(x, p) \\
&=\frac{1}{\pi} \int d x^{\prime} d p^{\prime} \exp [\left.-2 i\left(\lambda^{-2} x_{\theta} x_{\theta}^{\prime}+\lambda^{2} p_{\theta} p_{\theta}^{\prime}\right)\right] \\
& \times \exp \left[-\left(\lambda^{-2} x_{\theta}^{\prime 2}+\lambda^{2} p_{\theta}^{\prime 2}\right)\right] Q_{\lambda \theta}^{\mathrm{c}}\left(i x^{\prime}, i p^{\prime}\right)
\end{aligned}
$$

We see that the continuation of $Q_{\lambda \theta}$ to the purely imaginary part of complexified phase space contains an explicate description of the Wigner function. 
Eq. (20) also implies a convenient criterion for the existence and regularity properties of the generalised $s$-transform, in terms of the growth of $A_{\lambda \theta}^{\mathrm{c}}$ as one moves away from the real plane. Specifically: $A_{\lambda \theta}^{(s)}(x, p)$ exists as a tempered distribution if and only if $\exp \left[-\frac{1}{1+s}\left(\lambda^{-2} x_{\theta}^{2}+\lambda^{2} p_{\theta}^{2}\right)\right] A_{\lambda \theta}^{\mathrm{c}}(i x, i p)$ exists as a tempered distribution ( $x$ and $p$ both real).

In view of Eq. (8) the condition may be rephrased: the necessary and sufficient condition for $A_{\lambda \theta}^{(s)}(x, p)$ to exist as a tempered distribution is that

$$
\exp \left[\frac{s}{1+s}\left(\lambda^{-2} x_{\theta}^{2}+\lambda^{2} p_{\theta}^{2}\right)\right]\left\langle(-x,-p)_{\lambda \theta}|\hat{A}|(x, p)_{\lambda \theta}\right\rangle
$$

exists as a tempered distribution. In particular, the generalised $s$-transform exists as a tempered distribution whenever the operator $\hat{A}$ is local, in the sense that it takes localised wave packets into wave packets which are again localised, in the same region of phase space.

\section{Complementarity}

We conclude by showing how these considerations can be used to illustrate the concept of complementarity.

Suppose one took a classical system characterised by the classical distribution $\Gamma$ and measured the observables $x_{\theta}, p_{\theta}$ to accuracies $\pm \frac{\lambda}{\sqrt{2}}, \pm \frac{1}{\sqrt{2} \lambda}$ respectively. The probability distribution for the values of $x, p$ as calculated from the measured values of $x_{\theta}, p_{\theta}$ would be

$$
\rho_{\lambda \theta}(x, p)=\frac{1}{\pi} \int d x^{\prime} d p^{\prime} \exp \left[-\lambda^{-2}\left(x^{\prime}{ }_{\theta}-x_{\theta}\right)^{2}-\lambda^{2}\left(p^{\prime}{ }_{\theta}-p_{\theta}\right)^{2}\right] \Gamma\left(x^{\prime}, p^{\prime}\right)
$$

Comparing the defining equation for $Q_{\lambda \theta}$, Eq. (1), with this equation it can be seen that the only difference consists in the replacement of the classical distribution $\Gamma$ with the Wigner function $W$. It is natural to ask: can one infer that the underlying distribution is $W$ rather than $\Gamma$ from experimental determinations of the functions $Q_{\lambda \theta}$ ? The answer to this question is that the non-classical nature of the $Q_{\lambda \theta}$ does not become experimentally manifest for any single pair of values of $\lambda$ and $\theta$.

However, it does show up if one makes measurements for many different values of these parameters.

To see this, consider Eq. (13) for the case $\phi=\theta$ :

$$
\begin{array}{r}
Q_{\lambda \theta}(x, p)=\frac{1}{\pi^{\frac{3}{2}} \lambda} \int d x^{\prime} d p^{\prime} \exp \left[-\frac{1}{\lambda^{2}}\left(\left(x^{\prime}-x_{\theta}\right)^{2}+y^{\prime 2}\right)+2 i p_{\theta} y^{\prime}\right] \\
\times{ }_{\theta}\left\langle x^{\prime}-y^{\prime}|\hat{\rho}| x^{\prime}+y^{\prime}\right\rangle_{\theta}
\end{array}
$$

In the limit as $\lambda \rightarrow 0$

$$
\frac{1}{\sqrt{\pi} \lambda} \exp \left[-\frac{1}{\lambda^{2}}\left(x^{\prime}-x_{\theta}\right)^{2}\right] \approx \delta\left(x^{\prime}-x_{\theta}\right)
$$

and

$$
\frac{1}{\sqrt{\pi} \lambda} \exp \left[-\frac{1}{\lambda^{2}} y^{\prime}+2 i p_{\theta} y^{\prime}\right] \approx \exp \left[-\lambda^{2} p_{\theta}^{2}\right] \delta\left(y^{\prime}\right)
$$

Consequently

$$
Q_{\lambda \theta}(x, p) \approx \frac{\lambda}{\sqrt{\pi}} \exp \left[-\lambda^{2} p_{\theta}^{2}\right]_{\theta}\left\langle x_{\theta}|\hat{\rho}| x_{\theta}\right\rangle_{\theta}
$$

in the small $\lambda$ limit. An experimental determination of the functions ${ }_{\theta}\left\langle x_{\theta}|\hat{\rho}| x_{\theta}\right\rangle_{\theta}$ provides one with enough information for an approximate reconstruction of the quantum state [8, 9, 29, 30, 31]. It follows, that an experimental determination of 
the $Q_{\lambda \theta}(x, p)$ (with $x, p$ both real) also provides one with enough information for such a reconstruction, provided that the functions are known for sufficiently small $\lambda$, and sufficiently many different values of $\theta$.

As an example, consider the Fock state $|n\rangle=|n\rangle_{10}$. The $Q$-function is

$$
Q(x, p)=Q_{10}(x, p)=\frac{1}{2 \pi n !} E^{n} e^{-E}
$$

where $E=\frac{1}{2}\left(x^{2}+p^{2}\right)$ is the classical energy. Using Eqs. (16) and (17) one finds

$$
\begin{aligned}
Q_{\lambda \theta}(x, p)=\frac{\operatorname{sech} \eta|\tanh \eta|^{n}}{2^{n+1} \pi n !}\left|H_{n}\left(|\operatorname{cosech} 2 \eta|^{\frac{1}{2}} z_{\lambda \theta}\right)\right|^{2} & \\
& \times \exp \left[-\frac{1}{2} \operatorname{sech} \eta\left(\lambda^{-1} x_{\theta}^{2}+\lambda p_{\theta}^{2}\right)\right]
\end{aligned}
$$

where $\eta=\ln \lambda, z_{\lambda \theta}=\frac{1}{\sqrt{2}}\left(\lambda^{-1} x_{\theta}+i \lambda p_{\theta}\right)$ and $H_{n}$ is a Hermite polynomial. $Q_{10}$ is peaked about the classical orbit at $E=n$. The behaviour of $Q_{\lambda \theta}$ for other values of $\lambda, \theta$ is illustrated in Figures 1 and 2 .

The functions $Q_{\lambda \theta}$ are obtained from the Wigner function by smoothing it in various ways. Different smoothings extract different features of the information present in the Wigner function. No single function extracts all the information (in explicate form). If one wants a complete description, then it is necessary to consider the complete set of functions (or else to use the continuation to complex values of $x$ and $p$ ).

The Wigner function describes the state much more efficiently, using the single function $W$, instead of the two-parameter family of functions $Q_{\lambda \theta}$ (restricted to real values of their arguments). This is an important advantage of the Wigner function. However, it can also be a disadvantage since, precisely because it represents the information in a very compact form, the Wigner function can be hard to interpret. The function $Q_{\lambda \theta}$, by contrast, describes the result of making a retrodictively optimal determination of phase space location. As such it has an immediate physical interpretation, and the picture it presents is much easier to assimilate intuitively.

The functions $Q_{\lambda \theta}$ can be thought of as a set of alternative views, or perspectives of a single underlying object. They might be compared with a set of photographs of a building taken from various directions. The description provided by any single photograph is incomplete. In order to get an adequate impression of the building as a whole, one needs to see how it looks from every direction.

It appears to us that this discussion provides some additional insight into the intuition underlying Bohr's concept of complementarity. In his book Interpreting the Quantum World Bub 32 uses Escher's well-known print Waterfall as "a visual metaphor for a quantum world, in which the properties of physical systems 'fit together' in different classical or Boolean perspectives that cannot be put together into a single Boolean framework". The above considerations may be taken as an alternative illustration of what is, we believe, essentially the same point. Measurements performed for one particular pair of values of $\lambda, \theta$ are not sufficient to exclude the possibility that the observed probability distribution results from an underlying classical distribution $\Gamma$ via Eq. (22). It is only when one considers the complete set of functions $Q_{\lambda \theta}$ that one realises that they cannot all be accounted for in the same way, in terms of a single classical distribution $\Gamma$ (except in those cases where the Wigner function is itself a possible classical distribution [33]).

\section{REFERENCES}

[1] Wódkiewicz, K., 1984, Phys. Rev. Lett., 52, 1064. Also see Wódkiewicz, K., 1986, Phys. Lett. A, 115, 304; 1987, Phys. Lett. A, 124, 207. 
[2] Lalović, D., Davidović, D.M., and Bijedić, N., 1992, Phys. Rev. A 46, 1206; 1992, Physica $A, \mathbf{1 8 4}, 231$.

[3] Stoler, D., 1970, Phys. Rev. D, 1, 3217; 1971, ibid., 4, 1925; Yuen, H.P., 1976, Phys. Rev. A, 13, 2226; Hollenhorst, J.N., 1979, Phys. Rev. D, 19, 1669. For a review see Schumaker, B.L., 1986, Phys. Rep., 135, 317.

[4] Halliwell, J.J., 1992, Phys. Rev. D, 46, 1610.

[5] Wünsche, A., 1996, Quantum Semiclass. Opt., 8, 343.

[6] Wünsche, A., and Bužek, V., 1997, Quantum Semiclass. Opt., 9, 631.

7] Appleby, D.M., 1999, J. Mod. Opt. 46, 813 (also available as Los Alamos e-print quant$\mathrm{ph} / 9805028$.

8] Leonhardt, U., and Paul, H., 1995, Prog. Quant. Electr. 19, 89.

[9] Leonhardt, U., 1997, Measuring the Quantum State of Light (Cambridge: Cambridge University Press).

[10] Mehta, C. L., and Sudarshan, E. C. G., 1965, Phys. Rev., 138, B274.

[11] Cahill, K. E., and Glauber, R. J., 1969, Phys. Rev., 177, 1857; 1882.

[12] PrugovečKi, E., 1978, Ann. Phys. (N.Y.), 110, 102.

[13] Mizrahi, S.S., 1984, Physica A, 127, 241; 1986, Physica A, 135, 237; 1988, Physica A, 150, 541.

[14] Davidović, D.M., and Lalović, D., 1993, J. Phys. A, 26, 5099.

[15] Busch, P., Grabowski, M., and Lahti, P.J., 1995, Operational Quantum Physics (Berlin: Springer-Verlag).

[16] Leonhardt, U., and Paul, H., 1994, Phys. Rev. Lett., 72, 4086; 1994, J. Mod. Opt., 41, 1427; Poyatos, J.F., Walser, R., Cirac, J.I.. and Zoller, P., 1996, Phys. Rev. A, 53, R1966; BANASZEK, K., 1998, xxx.lanl.gov, quant-ph/9801059.

[17] O'Connell, R. F., and Wigner, E. P., 1981, Phys. Lett. A, 85, 121.

[18] Bohm, D., and Hiley, B.J., 1993, The Undivided Universe (London: Routledge).

[19] Menta, C.L., 1964, J. Math. Phys., 5, 677.

[20] Lalović, D., Davidović, D.M., and Bijedić, N., 1992, Phys. Lett. A 166, 99.

[21] LeE, H.W., 1995, Phys. Rep., 259, 147.

[22] Kano, Y., 1965, J. Math. Phys., 6, 1913.

[23] Bargmann, V., 1961, Commun. Pure and Appl. Math., 14, 187; 1962, Proc. Natl. Acad. Sci. U.S., 48, 199.

[24] Perelomov, A., 1986, Generalized Coherent States and Their Applications (Berlin: Springer-Verlag).

[25] Glauber, R.J., 1963, Phys. Rev., 131, 2766.

[26] Bell, J.S., 1964, Physics, 1, 195; 1987, Speakable and Unspeakable in Quantum Mechanics (Cambridge: Cambridge University Press).

[27] Bohr, N., 1934, Atomic Theory and the Description of Nature (Cambridge: Cambridge University Press); 1958, Atomic Physics and Human Knowledge (New York: John Wiley and Sons); 1963, Essays 1958-1962 on Atomic Physics and Human Knowledge (New York: John Wiley and Sons).

[28] Gradshteyn, I.S., and Ryzhik, I.M., 1980, Table of Integrals, Series, and Products, corrected and enlarged edition prepared by A. JEFFREY, incorporating the fourth edition prepared by Yu. V. Geronimus and M. Yu. Tseytlin (New York: Academic Press).

[29] Vogel, K., and Risken, H., 1989, Phys. Rev. A 40, 2847.

[30] Smithey, D.T., Beck, M., Raymer M.G., and Faridani, A., 1993, Phys. Rev. Lett. 70, 1244; Smithey, D.T., Beck, M., Cooper, J., and Raymer, M.G., 1993, Phys. Scr. T 48, 35; Beck, M., Raymer, M.G., Wamsley, I.A., and Wong, V., 1993, Opt. Lett. 18, 2041; Beck, M., Smithey, D.T., and Raymer, M.G., 1993, Phys. Rev. A 48, R890.

[31] Mancini, S., Man'Ko, V.I., and Tombesi, P., 1995, Quantum Semiclass. Opt., 7, 615; D'Ariano, G.M., Mancini, S., Man'Ko, V.I., and Tombesi, P., 1996, Quantum Semiclass. Opt., 8, 1017; Mancini, S., Man'ko, V.I., and Tombesi, P., 1997, Quantum Semiclass. Opt., $\mathbf{9}, 615$.

[32] Bub,J., 1997, Interpreting the Quantum World (Cambridge: Cambridge University Press).

[33] Hudson, R.L., 1974, Rep. Math. Phys., 6, 249; Soto, F., and Claverie, P., 1983, J. Math. Phys., 24, 97; Jagannathan, R., Simon, R., Sudarshan, E.C.G., and Vasudevan, R., 1987, Phys. Lett. A, 120, 161; Narcowich, F.J., 1988, J. Math. Phys., 29, 2036; Bröcker, T., and Werner, R.F., 1995, J. Math. Phys., 36, 62. 


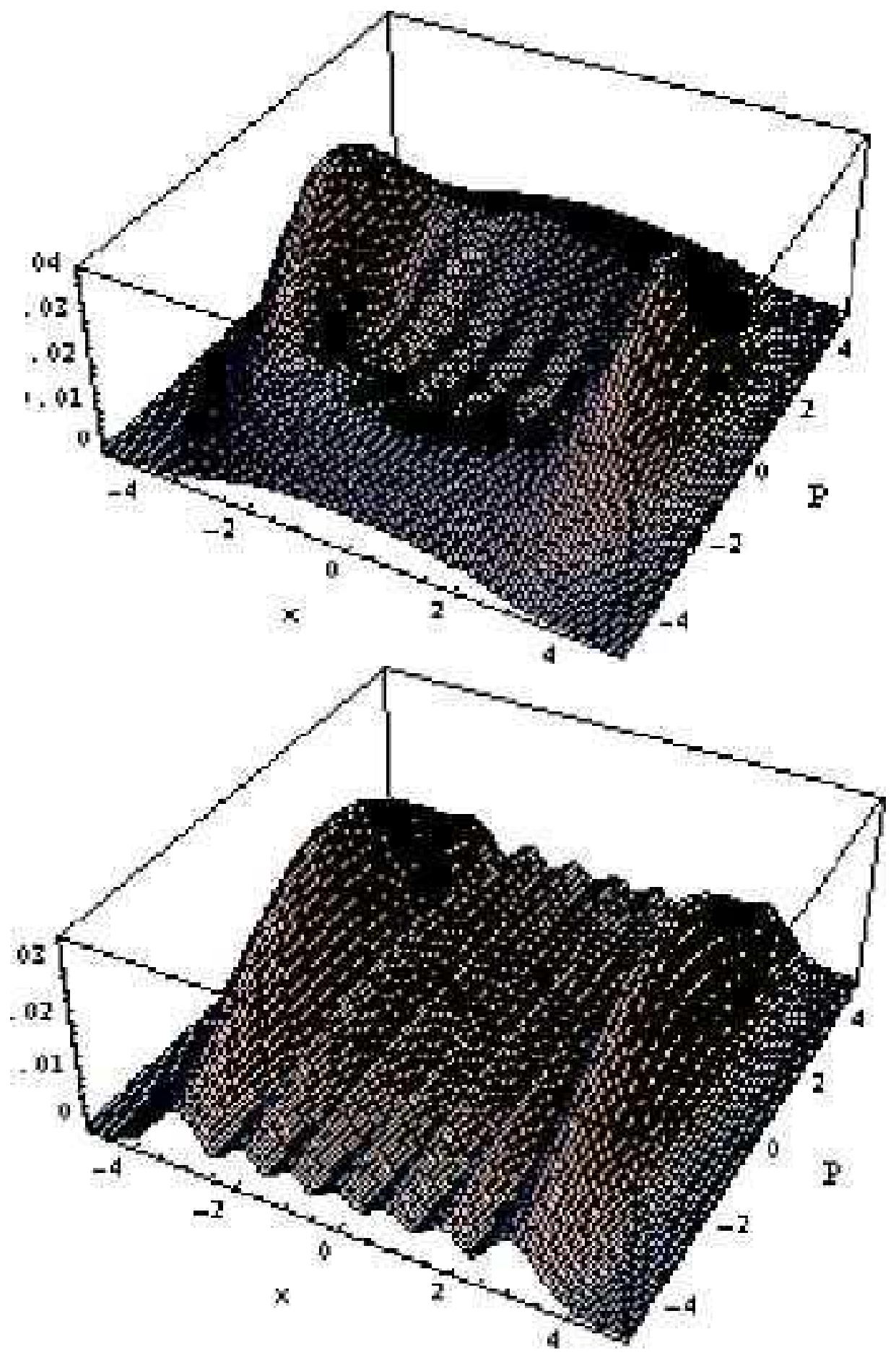

Figure 1. The function $Q_{\lambda \theta}$ for the state $|n\rangle_{10}$, with $n=6$ and $\theta=0$. In the top diagram $\lambda=0.5$. In the bottom diagram $\lambda=0.25$. See Eq. (25). It can be seen that at $\lambda=0.25$ the function is beginning to take its asymptotic form, as given by Eq. (23). 


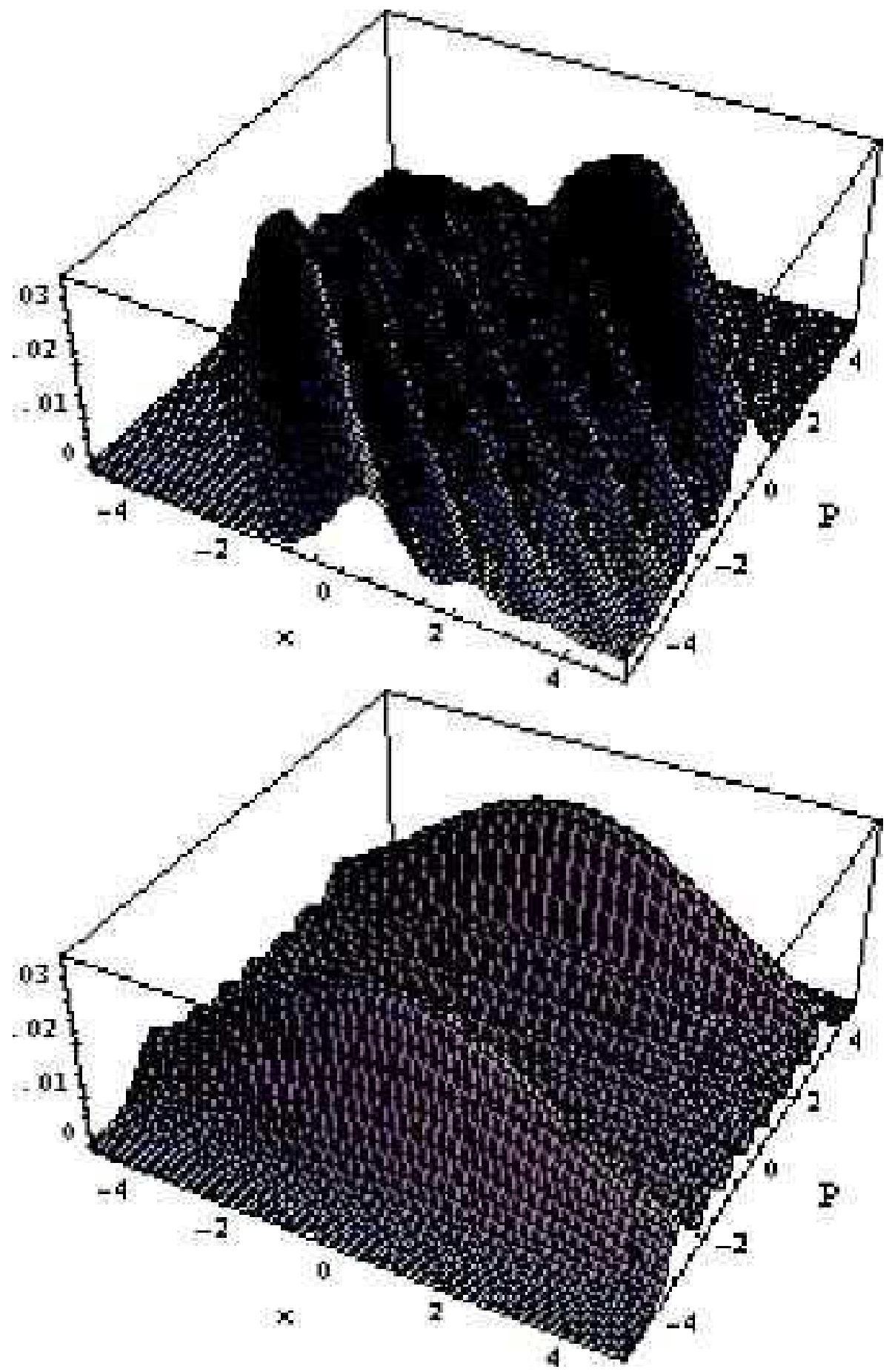

FIgURE 2. The function $Q_{\lambda \theta}$ for the state $|n\rangle_{10}$, with $n=6$ and $\lambda=0.25$. In the top diagram $\theta=\frac{\pi}{4}$. In the bottom diagram $\theta=\frac{\pi}{2}$. See Eq. (25). It can be seen that the effect of varying $\theta$ is simply to rotate the distribution. This is a consequence of the rotational symmetry of the state $|n\rangle_{10}$. 